BNL-95366-2011-CP

\title{
Application of Triple Coincidence for the Detection of Small Amounts of Special Nuclear Materials
}

\author{
I. Dioszegi, C. Salwen, and L. Forman \\ Presented at the $11^{\text {th }}$ International Conference on Applications of Nuclear Techniques \\ Crete, Greece \\ June 12-18, 2011
}

June 2011

\author{
Nonproliferation and National Security Department \\ Brookhaven National Laboratory
}

\section{U.S. Department of Energy \\ Office of Science; National Nuclear Security Administration}

\footnotetext{
Notice: This manuscript has been authored by employees of Brookhaven Science Associates, LLC under Contract No. DE-AC02-98CH10886 with the U.S. Department of Energy. The publisher by accepting the manuscript for publication acknowledges that the United States Government retains a non-exclusive, paid-up, irrevocable, world-wide license to publish or reproduce the published form of this manuscript, or allow others to do so, for United States Government purposes.

This preprint is intended for publication in a journal or proceedings. Since changes may be made before publication, it may not be cited or reproduced without the author's permission.
} 


\section{DISCLAIMER}

This report was prepared as an account of work sponsored by an agency of the United States Government. Neither the United States Government nor any agency thereof, nor any of their employees, nor any of their contractors, subcontractors, or their employees, makes any warranty, express or implied, or assumes any legal liability or responsibility for the accuracy, completeness, or any third party's use or the results of such use of any information, apparatus, product, or process disclosed, or represents that its use would not infringe privately owned rights. Reference herein to any specific commercial product, process, or service by trade name, trademark, manufacturer, or otherwise, does not necessarily constitute or imply its endorsement, recommendation, or favoring by the United States Government or any agency thereof or its contractors or subcontractors. The views and opinions of authors expressed herein do not necessarily state or reflect those of the United States Government or any agency thereof. 


\title{
Application of Triple Coincidence for the Detection of Small Amounts of Special Nuclear Materials
}

\author{
I. Dioszegi ${ }^{\mathrm{a}}$, C. Salwen ${ }^{\mathrm{a}}$, and L. Forman ${ }^{\mathrm{b}}$ \\ ${ }^{a}$ Brookhaven National Laboratory, Upton, New York 11973-500, USA \\ ${ }^{b}$ Ion Focus Technology Inc., Miller Place, New York 11764, USA
}

\begin{abstract}
We constructed a device that measures two $\gamma$-rays and one neutron from spontaneous fission and any resulting multiplication chains. It extends the associated particle technique based upon correlated counting of the multiplicity of gamma-rays and neutrons released in spontaneous- or neutron-induced fission. There are two advantages in incorporating a third detector in the design over the standard two-detector version. First, we found that random uncorrelated events dominate the background of coincident counting with a gamma-ray- and neutron-detector. These might be suppressed by requiring an additional coincidence. Second, the time history of gamma-ray emission between the two gamma-ray detectors is related to multiplication in the target media. Multiplication in highly enriched uranium is much greater than in depleted uranium.
\end{abstract}

Keywords: Special Nuclear Material, Spontaneous Fission.

PACS: 25.85.Ca, 29.40.Mc.

\section{INTRODUCTION}

We are developing ${ }^{1}$ a device that identifies Special Nuclear Material (SNM) by detecting spontaneous fission $\gamma$-rays and neutrons. Spontaneous fission emits several neutrons and $\gamma$-rays simultaneously, but also may exhibit a fast die-away component resulting from fission multiplication via chain reactions. In our device, we exploit a very short coincidence window to reduce the random background of $\gamma$-radiation coming from the target material's radioactive decay modes, and from the neutrons and $\gamma$-rays generated by cosmic rays. When there are large amounts of material nearby, cosmic-rays generate considerable numbers of fast neutrons whose energy distribution is similar to that of the fission neutrons (the so-called ship effect); therefore, filtering out these ship-effect neutrons is of the utmost importance. 


\section{TRIPLE COINCIDENCE DETECTION}

\section{The Experimental Device}

Our device (Fig. 1.) detects triple coincidences (TCs) of one neutron and (at least) two $\gamma$-rays emitted during the spontaneous fission event in a fast coincidence $(\sim 100 \mathrm{~ns}$ wide) window. The $\gamma$-detectors are fast $\mathrm{BaF}_{2^{-}}(5 \times 5 \times 20 \mathrm{~cm})$ and $\mathrm{LaBr}_{3}-(2 \times 2 ")$ scintillators, and the neutron detectors are large area $(30 \times 30 \times 5 \mathrm{~cm})$ plastic detectors. Data recorded in a list mode are read out by VME-based electronics made by CAEN (Italy), and then are processed and analyzed by LabVIEW routines.

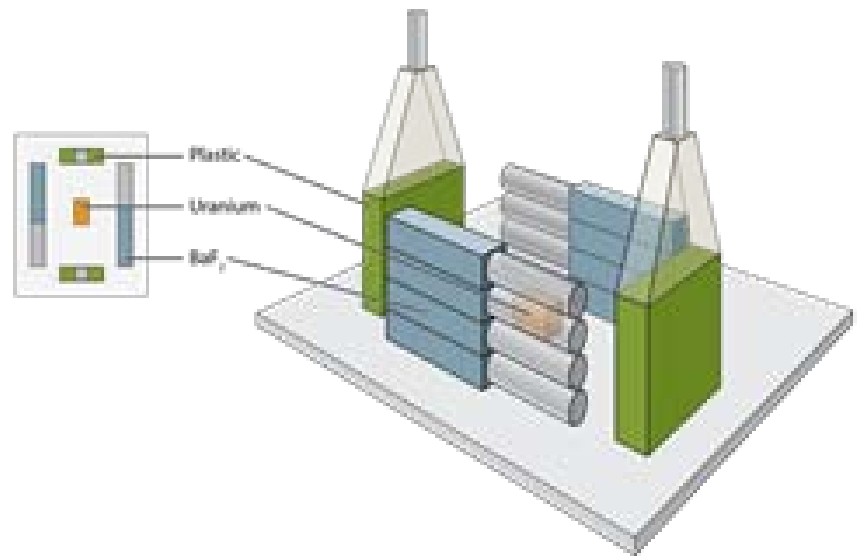

FIGURE 1. Arrangements of the Triple Coincidence Detector Large-area plastic detectors and two $\mathrm{BaF}_{2}$ scintillator walls surround the target material. In other variation of this setup, a $\mathrm{LaBr}_{3}$ detector replaced one of the plastic detectors.

We calibrated the detector system with a ${ }^{252} \mathrm{Cf}$ fission source. Fig. 2 presents the time of flight (TOF) spectra of the different detector groups.

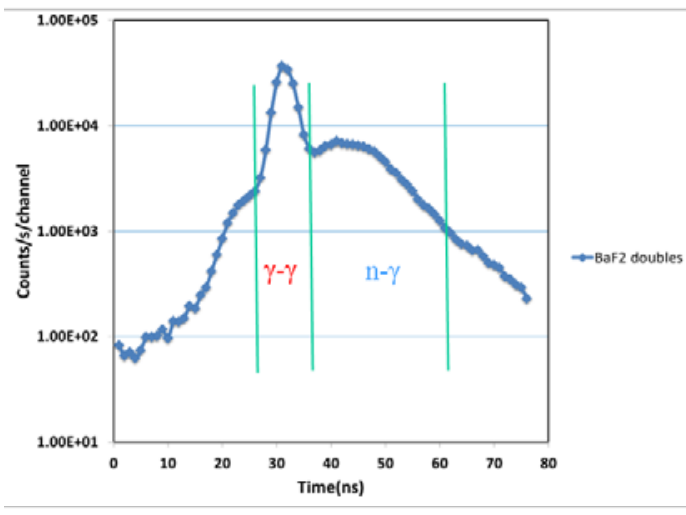

a)

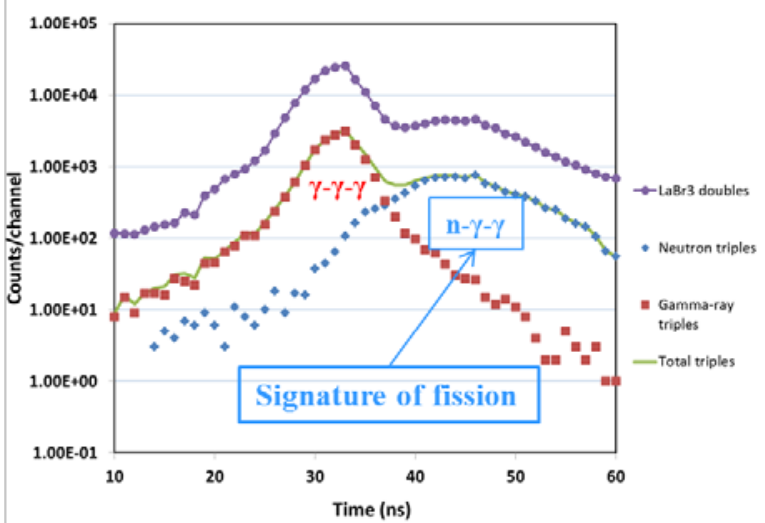

b)

FIGURE 2. ${ }^{252} \mathrm{Cf}$ time of flight (TOF) spectra of $\mathrm{BaF}_{2}$ - plastic (panel a)) and $\mathrm{LaBr}_{3}$ - plastic (panel b)). In panel b) the green line shows the $\mathrm{BaF}_{2}-\mathrm{LaBr}_{3}-$ plastic triple coincidences. For an explanation, see the text. 
The left panel (a) shows a TOF spectrum wherein the time is started by the $\mathrm{BaF}_{2}$ detectors and is stopped by the plastic ones; the right panel (b) shows the $\mathrm{LaBr}_{3}-$ plastic coincidence events. The green line in the latter depicts triple events that occur in each of the $\mathrm{BaF}_{2}-\mathrm{LaBr}_{3}$ - plastic detectors. The data analysis software extracted them from the recorded double-coincidence events. Applying time gates on the left panel (marked " $\gamma-\gamma$ " and " $n-\gamma$ ") separates the triple-coincidence events into " $\gamma-\gamma-\gamma$ " and " $n-\gamma-\gamma$ " triple coincidences, where the presence of the " $n-\gamma-\gamma$ " neutron triple coincidence is the signature of nuclear fission in the target.

When the triple event is correlated with fission, the plastic neutron detector records one of the fission neutrons, and the opposite $\gamma$-ray detector walls record the two coincidence fission $\gamma$-rays. However, there are other possibilities. The plastic detector also is sensitive to $\gamma$-rays, so resulting in $\gamma-\gamma-\gamma$ triple coincidences; however, these are distinguishable from $n-\gamma-\gamma$ events based on the neutrons' slower TOF. Also sometimes, an energetic $\gamma$-ray can be scattered from one wall of the detector to the other one, generating false coincidences. Again, we can discriminate them by their TOF. A third possibility especially is important if the target is a strong source of $\gamma$-ray singles, as is natural uranium that emits multiple $\gamma$-rays because of natural radioactive decay. In this case, the $\gamma$-ray singles generate a high random coincidence-rate in the detector system, with a uniform time-distribution.

\section{Understanding The Background}

To understand the background fully, we undertook several background measurements using fission- and radioactive-sources, and also acquired roombackground data. Figure 3 depicts a valid $\gamma$-n fission event (panel (a)) along with possible background scenarios. The blue detector is the plastic one; the red detectors are the $\gamma$-ray detectors. In panel (b) a source $\gamma$-ray is scattered from the $\gamma$-ray detector on to the neutron detector (a correlated source event). In panel (c) two independent source $\gamma$-rays hit the neutron and $\gamma$-detectors within the coincidence recording window (uncorrelated source event). Panel (d) shows a room background y-ray (could be a $\gamma$ ray originating from a cosmic ray, for example) scattered from one detector to the other (a correlated room background event); finally (e) illustrates two random $\gamma$-rays hitting two detectors within the coincidence recording window.

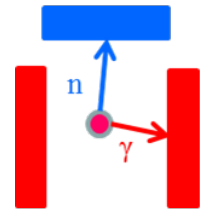

a)

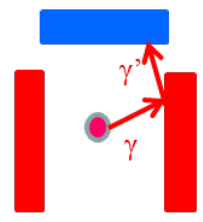

b)

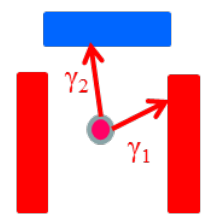

c)

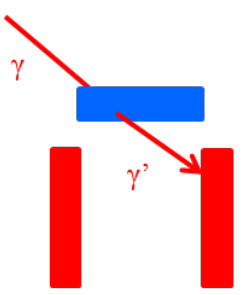

d)

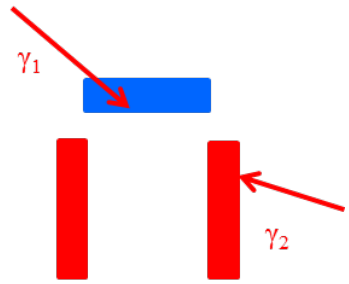

e)

FIGURE 3. Various scenarios; correlated-, random source-, and room background-ones. For explanation, see the text.

The various background events result in characteristic features in the onedimensional TOF spectra, but are easier to study n two-dimensional TOF-energy 
scatter plots. Figure 4 plots the recorded one-dimensional TOF spectra of room background (brown squares), of ${ }^{137} \mathrm{Cs}$ source background (diamonds)), and of two depleted uranium (DU) samples (3 kg, filled circles, and $11 \mathrm{~kg}$, triangles). The roombackground curve exhibits a big correlated peak (around 45 ns (relative) TOF) that is due to scenario (d) in Fig. 3. However, the $\gamma$-ray source, ${ }^{137} \mathrm{Cs}$, has a shoulder in addition to the prominent peak at $\sim 45$ ns position in the one-dimensional TOF spectrum. It is apparent, from the corresponding lower insert, , that this shoulder is a manifestation of the $662 \mathrm{keV}{ }^{137} \mathrm{Cs} \gamma$-line, wherein a random room-background radiation triggers the plastic detector. Consequently, this background component is constant in time. This effect is much stronger in the DU (upper inset), where the target emits multiple uncorrelated $\gamma$-rays, resulting in the horizontal bands seen in the twodimensional figure. Requiring a triple coincidence eliminates this strong random background.

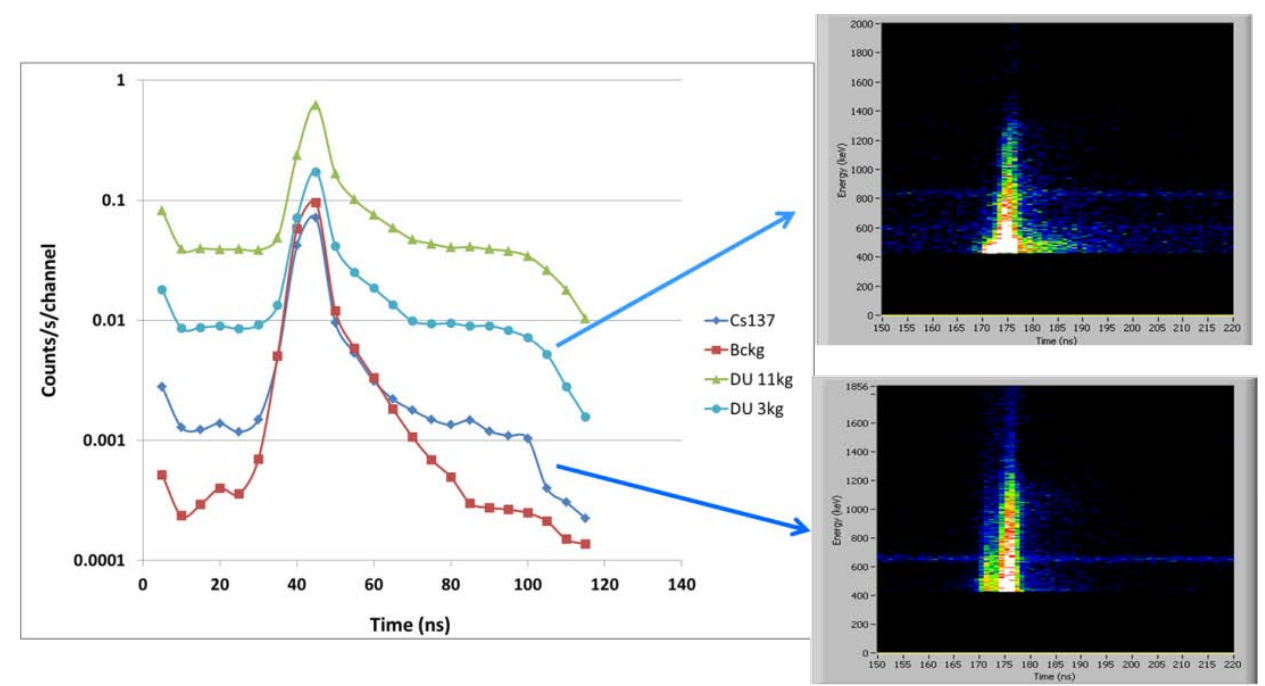

FIGURE 4. One-dimensional $\mathrm{LaBr}_{3}$ TOF spectra of different targets and room background. The insets show the two-dimensional Energy-TOF spectra for ${ }^{137} \mathrm{Cs}$ (lower insert), and $3 \mathrm{~kg}$ DU (upper insert).

The time scales in the inserts have a different (arbitrary) time offset.

\section{Triple Coincidences}

Table 1, below, gives explanations for the improvement gained in the peak-tobackground ratio using triple coincidences:

$R_{d c}$ is the correlated rate of $n-\gamma$ doubles from spontaneous fission source with a rate $\mathrm{S}_{\mathrm{fo}}$;

$R_{d u}$ is the uncorrelated rate of $n-\gamma$ doubles source with rate $S_{\gamma}$;

$v$ is the mean neutron (or $\gamma$, respectively) multiplicity of fission;

$\mathrm{D} \gamma_{1}(\gamma)$ is the response of detector \#1 to $\gamma$-rays;

$\mathrm{D}_{\mathrm{n}}(\mathrm{n})$ is the neutron-detector's response to neutrons;

$\Delta \mathrm{t}$ is the width of the coincidence window;

Similarly, this applies to triples with index " $t$ ". 
TABLE 1. Double- and triple-coincidence rates.

\begin{tabular}{lcc}
\hline & Doubles & Triples \\
\hline Correlated & $\mathbf{R}_{\mathrm{dc}}=\mathrm{S}_{\mathrm{fo}} \mathbf{v}_{\gamma} \mathbf{D} \gamma_{1}(\gamma) \mathbf{v}_{\mathrm{n}} \mathbf{D}_{\mathrm{n}}(\mathbf{n})$ & $\mathbf{R}_{\mathrm{tc}}=\mathbf{R}_{\mathrm{dc}}\left(\mathbf{v}_{\gamma}-\mathbf{1}\right) \mathbf{D} \gamma_{2}(\gamma)$ \\
Uncorrelated & $\mathbf{R}_{\mathrm{du}}=\mathrm{S}_{\gamma} \mathbf{D} \gamma_{1}(\gamma) \mathrm{S}_{\gamma} \mathbf{D}_{\mathrm{n}}(\gamma) \Delta \mathrm{t}$ & $\mathbf{R}_{\mathrm{tu}}=\mathbf{R}_{\mathrm{du}} \mathbf{S}_{\gamma} \mathbf{D} \gamma_{2}(\gamma) \Delta \mathrm{t}$ \\
\hline
\end{tabular}

The triple-coincidence requirement does not affect the correlated radiation beyond applying the second $\gamma$-ray detector's response (including the solid angle and intrinsic efficiency), plus a factor $v$-1, the latter being greater than one. However, for uncorrelated radiation, a strong additional limiting-factor applies; viz., the source's strength multiplied by the width of the coincidence window, $\Delta \mathrm{t}$. The effect of the triple-coincidence filter is demonstrated on Figure 5, where the ${ }^{252} \mathrm{Cf}$ doubles show a signal- to-background ratio of 15 (in the $n-\gamma$ region), whereas this ratio is 150 for the $\mathrm{n}-\gamma-\gamma$ triples.

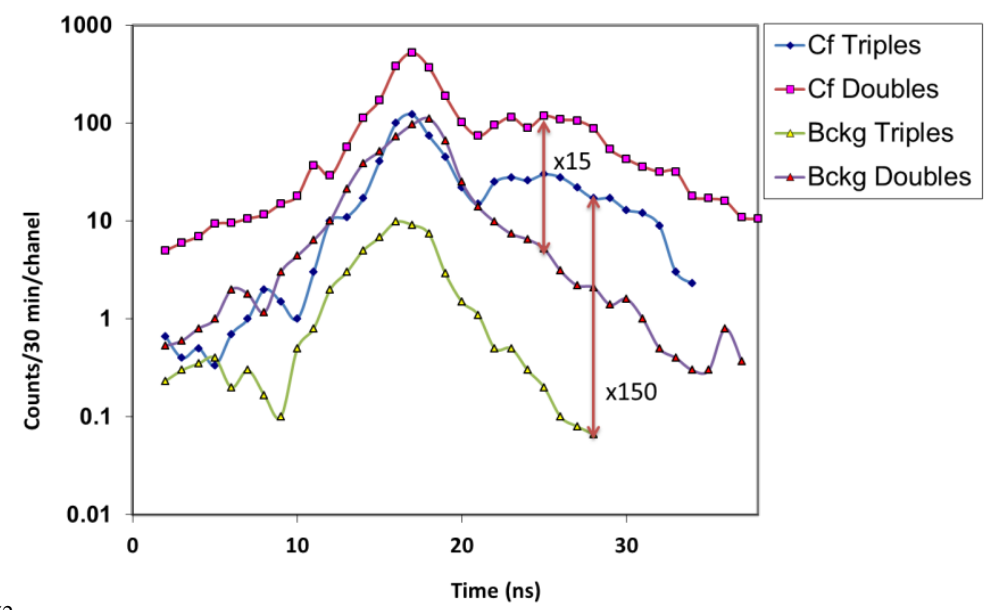

FIGURE 5. ${ }^{252} \mathrm{Cf}$ doubles and triples along with their corresponding background doubles and triples. There is an order-of-magnitude increase in the signal-to-background ratio when using triple- rather than double-coincidences.

\section{Triple Coincidences in Depleted Uranium}

Our experimental results from depleted uranium (DU) samples demonstrate the advantage of our triple-coincident technique. Figure 6 presents the triple-coincidence TOF spectra for 3- and 11-kg DU material, along with the ${ }^{137} \mathrm{Cs}$ radioactive source and room background shown as doubles in figure 4. We observed a substantial increase in counts in the $n-\gamma-\gamma$ region that we attribute to $n-\gamma-\gamma$ correlated events due to spontaneous fission in DU.

The correlated $\gamma$ background peak (at about $40 \mathrm{~ns}$ ) does not change significantly when going from room background to ${ }^{137} \mathrm{Cs}$ and the different quantities of DU (the spectra are normalized to the same measurement time), whereas the spectra of the doubles (figure 4) differ by an order-of-magnitude. This is because the energy of the backscatter of the source photon (figure 3, panel b), by then has fallen below the threshold of the detector's electronics. The forward scatter of a background photon 
(figure 3, panel d), may be sufficiently energetic in the backward angle to be recorded. The correlated background is target independent; it is due to the ever-present room background.

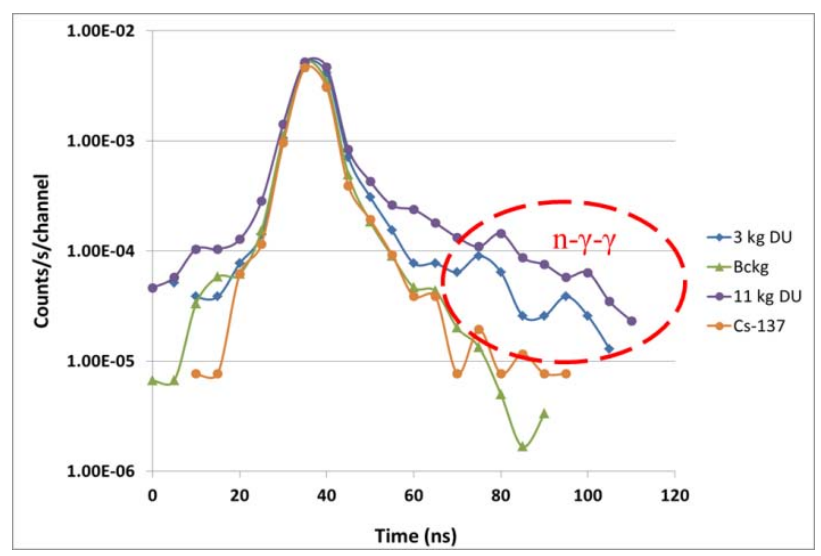

FIGURE 6. Triple-coincidence measurements of DU targets (3- and 11-kg) along with the ${ }^{137} \mathrm{Cs}$ and room backgrounds. The excess in the DU spectra in the $n-\gamma-\gamma$ region reflects the spontaneous fissions correlated $n-\gamma-\gamma$ emissions.

To verify that the observed enhancement in counts is due to fission-neutron events, we calculated the energy of the neutrons from their time-of-flight. The calculated energy spectrum for the $11 \mathrm{~kg}$ DU sample is displayed in this figure, along with the ${ }^{252} \mathrm{Cf}$ measured energy-spectrum, and also the normalized Maxwell distributions. These measured distributions show a characteristic Maxwellian energy distribution and they scale with the strength of the known source (for DU we use 2.0136 neutrons/g s, whereas that of the ${ }^{252} \mathrm{Cf}$ source was known to emit 4200 neutrons/sec). There are complicating factors, such as the significant multiplication in the DU, as predicted by spherical MCNP calculations (the DU samples were discs), and the many $\gamma$-rays emitted in the DU may be absorbed in the target. Moreover, inelastic neutronscattering in the DU will create a population of neutrons with lower energies, as are observed in the DU spectra.

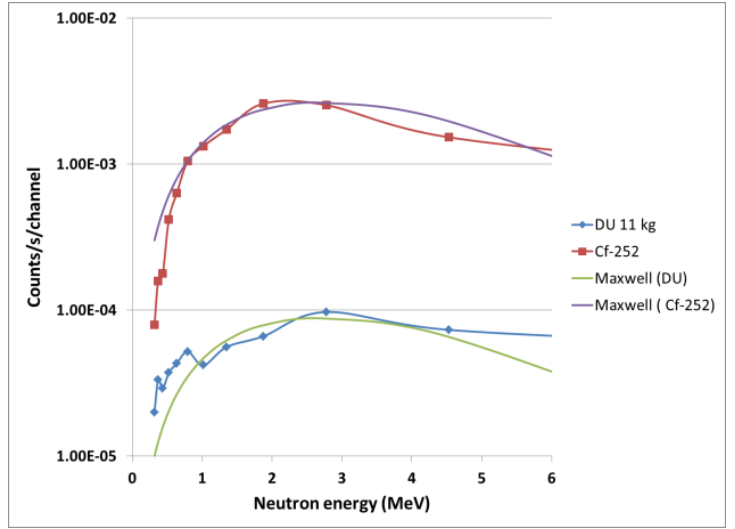

FIGURE 7 Measured distributions of fission-neutron energy of ${ }^{252} \mathrm{Cf}$ and $11 \mathrm{~kg}$ DU. The Maxwellian energy distributions scale well with the known fission rates of the source and the sample. 


\section{Triple Coincidences in Highly Enriched Uranium (HEU)}

We demonstrated that our method is sufficiently sensitive to detect DU. The required measurement time using our experimental setup was about 10 hours, which might be reduced considerably by using more detectors. Since the main goal of our project is detecting SNMs, we must estimate how long it would take to detect them. In our research described in this paper, we concentrated primarily on detecting highly enriched uranium. We employed $\mathrm{MCNPX}^{3}$ calculations to estimate the fission neutron rate of HEU- and DU-samples. Although the ${ }^{235} \mathrm{U}$ spontaneous fission rate is negligible, the fission neutrons from the (ever-present) ${ }^{238} \mathrm{U}$ spontaneous fissions induce ${ }^{235} \mathrm{U}$ fission, and furthermore, the ${ }^{235} \mathrm{U}$ fissions also undergo multiplication. Figure 8 presents the calculated number of fission neutrons as a function of mass for DU and HEU.

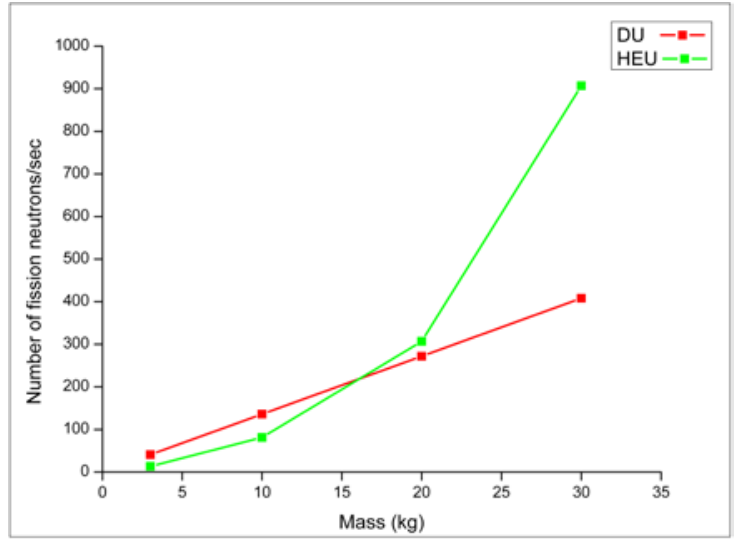

FIGURE 8. MCNPX prediction of the number of fission neutrons for various masses of DU and HEU. In HEU, we observe a large increase in neutron output above $20 \mathrm{~kg}$ due to fission multiplication.

The increase of numbers of fission neutrons in HEU is accompanied by a similar increase in the number of $\gamma$-rays (not shown here). Combining them, we expect a decrease in measurement time when assessing HEU compared to DU.

To differentiate between HEU and DU, we plan to utilize an additional effect. The extent of fission multiplication in DU is limited. In HEU, however, depending on its amount, this multiplication could be a strong, distinguishing factor. Thus, by detecting two $\gamma$-rays from the same fission event in DU, or two consecutive $\gamma$-rays from separate fission events during a fission chain in HEU, we can discriminate between HEU and DU.

Figure 9 depicts the measured relative $\gamma$-ray rate as a function of time in ${ }^{252} \mathrm{Cf}$ and DU, along with MCNPX calculations for DU and HEU. The measured background die-away time where there is no multiplication, reflects the time-resolution of the present experimental setup. The DU plotted therein shows a somewhat longer dieaway time. The MCNPX calculations also presented in the figure demonstrate that HEU has a substantially longer fission multiplication die-away time than would DU, thus making feasible their differentiation based on this parameter. 


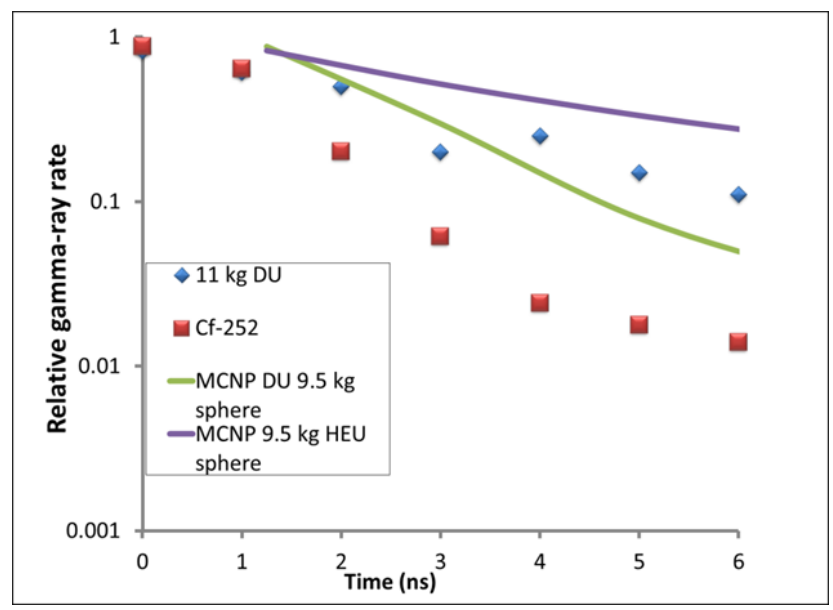

Figure 9. Triple gamma-ray time distribution compared to calculations.

\section{SUMMARY}

We developed a method for assessing and identifying SNMs, based on triple $n-\gamma-\gamma$ coincidence measurements. The success of our approach rests on the greatly improved signal-to-background ratio when requiring a second $\gamma$ coincidence. We demonstrated that fission multiplication in HEU results in a higher fission rate of neutrons and $\gamma$ rays, thus lowering the necessary measurement time possibly by an order-ofmagnitude. The die-away time of fission $\gamma$-rays is a potential indicator of presence of $\mathrm{HEU}$ due to the high fission multiplication rate in ${ }^{235} \mathrm{U}$.

\section{ACKNOWLEDGMENTS}

The present work has been supported by the U. S. Department of Energy (NA-22).

\section{REFERENCES}

1. L. Forman, I. Dioszegi and P. E. Vanier, Fast time correlation detection in associated particle spectrometry of spontaneous fission neutrons and cosmic-ray backgrounds. SPIE Proceedings Vol. 7805, Hard X-ray, Gamma-Ray and Neutron Detector Physics XII, San Diego, CA, 27 August 2010.

2. K. Shultis and R. E. Faw, Radiation Shielding, p. 82. Prentice Hall PTR, Upper Saddle River, NI 07458, 1996.

3. D. Pelowitz (Ed.), MCNPX User's Manual Version 2.6.0, Los Alamos National Laboratory Report, LA-CP-07-1473 (April 2008). 